\title{
Selection of glucocorticoid-sensitive patients in interstitial lung disease secondary to connective tissue diseases population by radiomics
}

This article was published in the following Dove Press journal:

Therapeutics and Clinical Risk Management

\begin{abstract}
Ding-Yun Feng, 1,* Yu-Qi Zhou, ', Yan-Fang Xing, ${ }^{2, *}$ Chuang-Feng $\mathrm{Li}^{3}$ Qing Lv, ${ }^{4}$ Jie Dong, ${ }^{5}$ Jie Qin, ${ }^{3}$ Yue-Fei Guo, ${ }^{3}$ Nan Jiang, ${ }^{6}$ Chencui Huang, ${ }^{7} \mathrm{Hai}-\mathrm{Tao} \mathrm{Hu},{ }^{8}$ Xing-Hua Guo, ${ }^{9}$ Jie Chen, ${ }^{10}$ Liang-Hong Yin, ${ }^{11}$ Tian-Tuo Zhang, ' Xing $\mathrm{Li}^{12}$

'Department of Respiration, The Third Affiliated Hospital of Sun Yat-sen University, Guangzhou 510630, People's Republic of China; '2Department of Nephrology, The Third Affiliated Hospital of Guangzhou Medical University, Guangzhou of Guangzhou Medical University, Guangzhou 510150 , People's Republic of China; ${ }^{3}$ Departmen of Radiology, The Third Affiliated Hospital of Su Yat-sen University, Guangzhou 510630 , People's
Republic of China; ${ }^{4}$ Department of Rheumatology, Republic of China; ${ }^{4}$ Department of Rheumato
The Third Affiliated Hospital of Sun Yat-sen The Third Affiliated Hospital of Sun Yat-sen
University, Guangzhou 510630, People's Republic University, Guangzhou 510630 , People's Republic
of China; ${ }^{5}$ Department of Radiotherapy, The Third Affiliated Hospital of Sun Yat-sen University, Guangzhou 5 10630, People's Republic of China; ${ }^{6}$ Department of Hepatic Surgery, The Third Affiliated Hospital of Sun Yat-sen University, Guangzhou 510630, People's Republic of China; ${ }^{7}$ The Associated Laboratory for Al, Cross-strait TsingHua Research Institute and Huiying Medical Technology, Dongsheng Science and Technology Park, HaiDian District, Beijing 100192, People's Republic of China; ${ }^{8}$ Department of Surgery, ChanCheng District Center Hospital, Foshan 528000 , People's Republic of China; 'Department of Rheumatology, The LingNan Hospital of Sun of Rheumatology, The LingNan Hospital of Sun Yat-sen University, Guangzhou 510000, People's Republic of China; ${ }^{10}$ Department of Oncology, People's Republic of China; 'Department of People's Republic of China; "Department of University, Guangzhou 510630, People's Republic of China; '2Department of Medical Oncology and Guangdong Key Laboratory of Liver Disease, The Third Affiliated Hospital of Sun Yat-sen University, Guangzhou 5 10630, People's Republic of China

*These authors contributed equally to this work
\end{abstract}

Correspondence: Xing Li

Department of Medical Oncology and Guangdong Key Laboratory of Liver Disease, The Third Affiliated Hospital of Sun Yat-sen University, 600 Tianhe Road, Guangzhou 510630, People's Republic of China Tel +862085252212

Email lixing9@mail.sysu.edu.cn

Tian-Tuo Zhang

Department of Respiration, The Third Affiliated Hospital of Sun Yat-sen University, 600 Tianhe Road Guangzhou 510630, People's Republic of China Tel +862085252212

Email zhtituli@।63.com
Purpose: The effect of glucocorticoid(s) on connective tissue disease (CTD)-related interstitial lung disease (ILD) is controversial. This multicenter study aimed to identify glucocorticoidsensitive patients using a radiomics approach.

Methods: A total of 416 CTD-ILD patients who began glucocorticoid treatment at the discretion of the attending physician, with or without cyclophosphamide, were included in this study. High doses were defined as pulsed intravenous methylprednisolone, an initial dose of 1 $\mathrm{mg} / \mathrm{kg} /$ day of prednisolone or $0.8 \mathrm{mg} / \mathrm{kg} /$ day of methylprednisolone. Low doses were defined as those less than high doses. Radiomics features were manually extracted from primary lung lesions delineated on computed tomography images, and selected by variance, univariate feature selection, and least absolute shrinkage and selection operator regression model. The prediction models were developed using data from 309 patients from two centers and externally validated in 107 patients from four other hospitals.

Results: Treatment response in the training and validation groups was $38.5 \%$ and $36.4 \%$, respectively. Eleven radiomics features were selected from 1,029 features with predictive value. Random forest models built for radiomics features to predict treatment response yielded a sensitivity of 0.897 . The calibration curve of a nomogram demonstrated good agreement between prediction and observation. Decision curve analysis indicated that glucocorticoid was beneficial if the predicted response rate was $50 \%-60 \%$ for an individual. High doses of glucocorticoids and cyclophosphamide yielded superior efficacy.

Conclusion: Radiomics-based predictive models reliably identified glucocorticoid-sensitive CTD-ILD patients. Short-term, high-dose glucocorticoid with cyclophosphamide yielded promising results as a potential therapy.

Keywords: radiomics, connective tissue diseases, interstitial lung diseases, glucocorticoid

\section{Plain language summary}

This multicenter study developed radiomics-based predictive models to assess the response to glucocorticoid treatment of connective tissue disease (CTD)-related interstitial lung disease (ILD). The radiomics-based predictive system was built using data from patients from two centers and was externally validated in patients from four other hospitals, which indicated that this technology yielded reasonable universality. An optimal glucocorticoid dose (pulsed intravenous methylprednisolone, an initial dose of $1 \mathrm{mg} / \mathrm{kg}$ /day of prednisolone or $0.8 \mathrm{mg}$ / $\mathrm{kg}$ /day of methylprednisolone) was identified, which may be used as guidance for future clinical practice in CTD-ILD. Glucocorticoid doses for each patient were not identical because this was not a clinical trial. 


\section{Introduction}

Interstitial lung diseases (ILD) are a heterogeneous group of disorders characterized by inflammation and/or fibrosis of the lungs, varying from idiopathic interstitial pneumonias to secondary variants, including ILD-associated connective tissue diseases (CTD). ${ }^{1}$ ILD with fibrosis is often incurable and is associated with significant morbidity and mortality. ${ }^{2}$ Treatments for CTD-ILD have been controversial and lack widely acknowledged supportive evidence. Glucocorticoid, cyclophosphamide, azathioprine, and mycophenolate mofetil have been the most extensively studied agents. ${ }^{3,4}$ Among these, glucocorticoids are widely used in the management of CTD-ILD, but with little clinical trial-based evidence and no consensus regarding the route of administration, dosage, and course. ${ }^{5,6}$ Nevertheless, the treatment efficacy of glucocorticoids is far from satisfactory. ${ }^{7}$ Because longterm and high-dose glucocorticoid treatment usually results in severe side effects, ${ }^{8}$ identifying patients who are likely to respond to glucocorticoids would be a potential solution to improve prognosis.

Notably, advances in image analysis algorithms and radiomics have made it possible to reproducibly and automatically quantify imaging phenotypes by extracting a large number of image features. ${ }^{9}$ Radiomics has attracted increased attention in recent years and offers prognostic and diagnostic value in multiple diseases, especially malignancies. ${ }^{10,11}$ Radiomics converts medical images into high-dimensional, mineable data via high-throughput extraction of quantitative features, followed by subsequent data analysis for decision support. Image analysis provides information that cannot be identified by the naked eye. This process of radiomics-based stratification can provide a far more detailed characterization of phenotype than current criteria, which may lead to a new era in defining CTD-ILD. The target of CTD-ILD stratification should be treatment guidance, in which radiomics has a promising role. However, radiomics has yet to be applied to ILD.

In this multicenter study, we investigated engineered lesion radiomics features extracted from high-resolution computed tomography (HRCT) images and tested these features against the efficacy of glucocorticoid with or without cyclophosphamide. Predictive models based on radiomics features and clinical data were constructed using a training cohort of patients from two institutions and subsequently validated in patients from four other hospitals. The study aimed to develop a practical methodology to predict treatment response to glucocorticoids for future clinical applications.

\section{Materials and methods Study population}

Six hospitals participated in this study. Patients from the Third Affiliated Hospital of Sun Yat-sen University and the Third Affiliated Hospital of Guangzhou Medical University (Guangzhou, Guangdong, China) comprised the training group. Patients from ChanCheng District Center Hospital, The LingNan Hospital of Sun Yat-sen University, Hengyang City Center Hospital, and The First Affiliated Hospital of JINAN University comprised the validation group. CTDILD was diagnosed based on clinical and HRCT data ${ }^{12}$ after discussion involving a multi-disciplinary diagnosis group consisting of respiratory physicians and thoracic radiologists. A total of 572 patients, who were initially diagnosed with ILD with stable extra-thoracic manifestations between June 2013 and September 2017, were evaluated as the training group; 309 patients who were administered glucocorticoid were included in this study. Of the 220 patients in the validation group, 107 were included in this study. A total of 376 patients (training group, $n=263$; validation group, $\mathrm{n}=113$ ) were excluded for the following reasons: idiopathic pulmonary fibrosis; acute infections confirmed by clinical symptoms; routine bacterial and viral bronchoalveolar lavage fluid antibody titers and cultures for pathogenic microorganisms; ILD due to tumor; drug side effects due to ILD medications; pneumoconiosis, pulmonary alveolar proteinosis; lack of HRCT data; and repeated administration (Figure 1). Before radiomics analysis, the diagnosis was independently re-evaluated according to consensus of three experienced diagnostic groups consisting of a thoracic radiologist and an experienced pulmonary specialist (YFG and YQZ; JQ and TTZ; CFL and DYF) on different occasions. The evaluations were performed in random order and the evaluators were unaware of the clinical diagnosis. This retrospective study was conducted in compliance with institutional policies to protect patient privacy and was approved by the institutional review board of the hospitals that participated in this study. Written informed consent was obtained from all patients.

\section{Treatment and follow-up}

Glucocorticoid treatment was administered as pulsed intravenous methylprednisolone, $0.75-1 \mathrm{mg} / \mathrm{kg} /$ day of prednisolone or $0.6-0.8 \mathrm{mg} / \mathrm{kg} /$ day of methylprednisolone). Glucocorticoid doses were stratified into high and low groups. High dosages were defined as pulsed intravenous methylprednisolone, an initial dose of $1 \mathrm{mg} / \mathrm{kg} /$ day of prednisolone or $0.8 \mathrm{mg} / \mathrm{kg} /$ day 


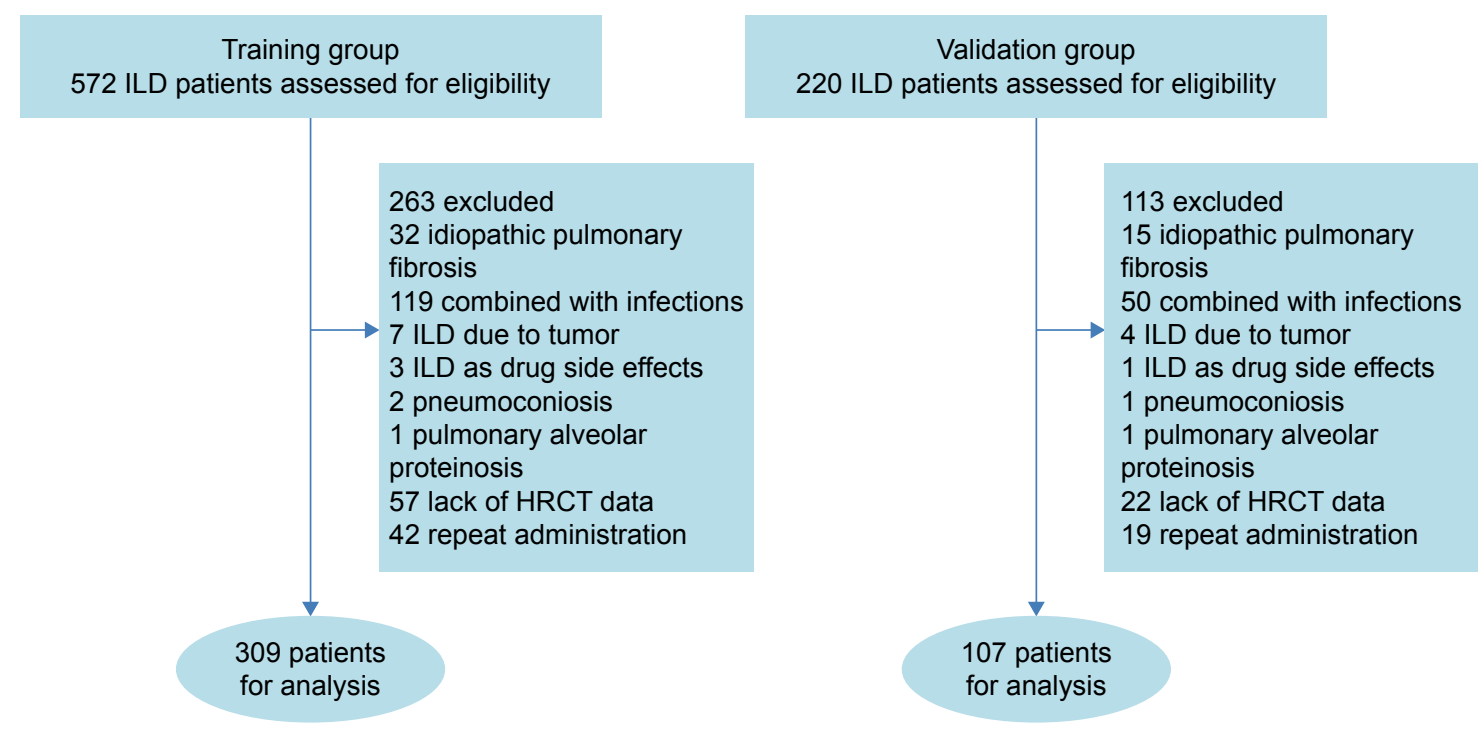

Figure I Screening of CTD-ILD patients.

Abbreviations: HRCT, high-resolution computed tomography; ILD, interstitial lung disease; CTD, connective tissue diseases.

of methylprednisolone. Low dosages were defined as an initial dose of $<1 \mathrm{mg} / \mathrm{kg} /$ day of prednisolone or $<0.8 \mathrm{mg} /$ $\mathrm{kg}$ /day of methylprednisolone. All patients were administered oral prednisolone or methylprednisolone for at least 1 month (median 5 months). All patients started or increased dosage of glucocorticoid with or without cyclophosphamide at the discretion of the attending physician. Disease-modifying anti-rheumatic drugs used before diagnosis of ILD were not changed before the response to treatment was assessed.

\section{Assessment of treatment response}

Clinical response was assessed according to symptoms and HRCT data 2-6 weeks after initial administration of glucocorticoid. Treatment response was defined by comparison with the condition before treatment: respiratory symptoms had improved and the radiographic lesions decreased by $>10 \%$ for at least 3 months. ${ }^{13}$ To investigate the long-term treatment response, a proportion of patients underwent assessment after 3-6 months of follow-up. Pulmonary function tests (PFTs) were performed at baseline and at 3-6 months. The three experienced diagnostic groups independently evaluated all forced vital capacity results for adequacy and repeatability, according to criteria from the American Thoracic Society. ${ }^{14}$

\section{Data collection}

HRCT was performed according to the standard clinical scanning protocols at the institutions and without intravenous contrast. The slice thickness ranged from 1 to $5 \mathrm{~mm}$ (median, $3 \mathrm{~mm}$ ).

\section{Patient and public involvement}

The patients and public were not involved in the design and conduct of this study.

\section{Radiomics quantification}

HRCT imaging phenotypes were described using a set of quantitative radiomics features extracted from the segmented lesion regions on the computed tomography (CT) scans. Briefly, CT images and lesion contours were imported into Radcloud (Huiying Medical Technology Co., Ltd, Beijing, China). Due to differences in pixel spacing and slice thickness, the image and lesion contours were subsequently normalized to isometric voxels $(3 \mathrm{~mm})$ using cubic interpolation. The primary lung lesions were manually delineated on the $\mathrm{CT}$ images by two independent thoracic radiologists (CFL and YFG) (Figure 2A). All contours were reviewed by the senior radiologist (JQ). All discrepancies were discussed by the three thoracic radiologists and two respiratory specialists. Subsequently, feature extraction was performed using an in-house developed Radiomics plug-in for 3DSlicer. A total of 1,029 radiomics features were included. The primary feature clusters were as follows: a graylevel size zone (GLSZM) quantifies gray-level zones in an image. A gray-level zone is defined as the number of connected voxels that share the same gray-level intensity. A gray-level run length matrix (GLRLM) quantifies gray-level runs, which are defined as the length in number of pixels and consecutive pixels that have the same gray-level value. The variability of graylevel intensity (GLN) values in the image was measured, with a lower value indicating more homogeneity in intensity values. Cluster shade is a measure of the skewness and uniformity 
of the gray level co-occurrence matrix (GLCM). A higher cluster shade reflects greater asymmetry in the mean. Cluster prominence is a measure of the skewness and asymmetry of the GLCM. A higher value reflects more asymmetry, and a lower value indicates a peak near the mean value and less variation around the mean. Large Area Emphasis (LAE) is a measure of the distribution of large area size zones, with a greater value indicative of larger size zones and more coarse textures. Long run high gray-level run emphasis (LRHGLRE) measures the joint distribution of long-run lengths with higher gray-level values. Overall, the primary features contained 19 first-order, 15 shape, 27 GLCM and 16 GLRLM features, and 16 GLSZM values. The secondary features were exponential, square, square root, logarithm, and wavelet transformation of the primary features.

\section{Data analysis}

To build the radiomics model, the following steps were performed in the training group and the models were tested in the validation group. Features with variance $<0.8$ were excluded from further analysis. Then, the associations between the imaging phenotype and the treatment response in the integrated dataset were analyzed using ANOVA, a method of univariate feature selection. Features were removed when $P$-values exceeded 0.05 . In the third step, the least absolute shrinkage and selection operator (LASSO) method, which is suitable for the regression of high-dimensional data, ${ }^{15}$ was used to select the most useful predictive features from the primary data set. The minimum mean square error was computed using a cross-validation method with five folds. According to the error, the best penalty parameter alpha of LASSO was obtained. Finally, features with nonzero coefficients were screened out for model training.

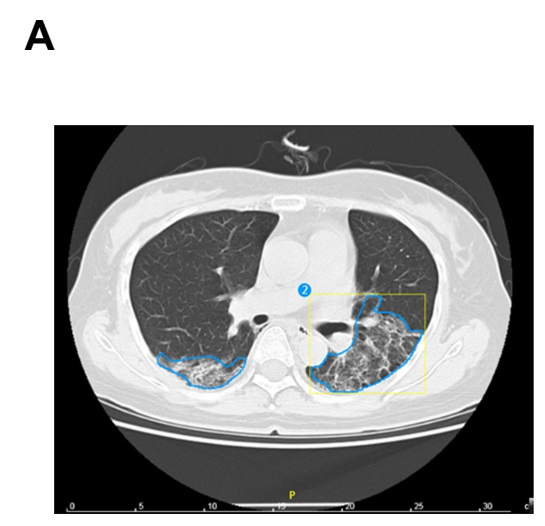

Figure 2 (Continued)

\section{Development of an individualized prediction model}

A random forest (RF) method ${ }^{16}$ and k-nearest neighbors (k-NN) were applied to develop radiomics-based diagnostic models for treatment using the training cohort. Furthermore, clinical factors in features were added to build new models. Four models were evaluated according to the area under the receiver operating characteristic (ROC) curve (AUC). A radiomics score (Rad-score) was calculated for each patient via a linear combination of selected features and weighted according to their respective coefficients. ${ }^{15}$ To provide the clinician with a quantitative tool to predict individual probability of treatment response, a nomogram was built on the basis of multivariable logistic analysis in the training group. For the nomogram, a radiomics score was calculated via a linear combination of selected radiomics features whose weights were dependent on logistic regression. The logistic regression was based on clinical factors and radiomics signatures were transformed to a nomogram.

\section{Clinical use}

To ascertain the clinical value of the nomogram, a decision curve was plotted to quantify the net benefits at different threshold probabilities in the validation dataset. ${ }^{15}$

\section{Results}

\section{Clinical characteristics}

Patient characteristics in the training and validation cohorts were similar except for primary diseases. Patients in the training group presented with more undifferentiated CTD $(29.1 \%$ vs $3.7 \%)$ and less rheumatoid arthritis (6.8\% vs $46.7 \%) ; 22.3 \%$ and $18.7 \%$ patients received high doses of

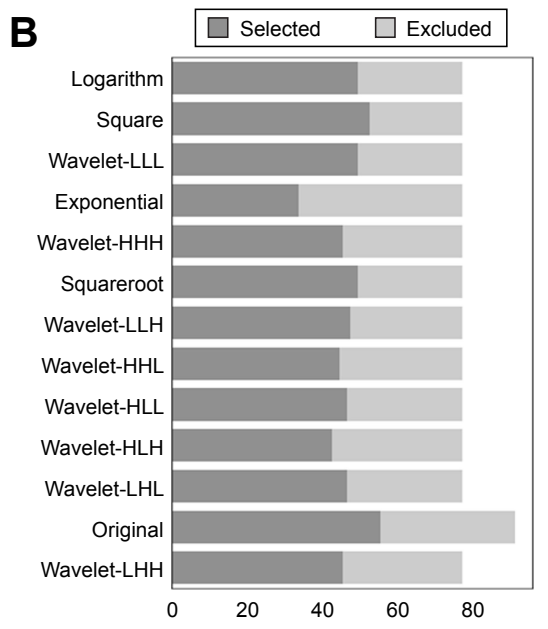




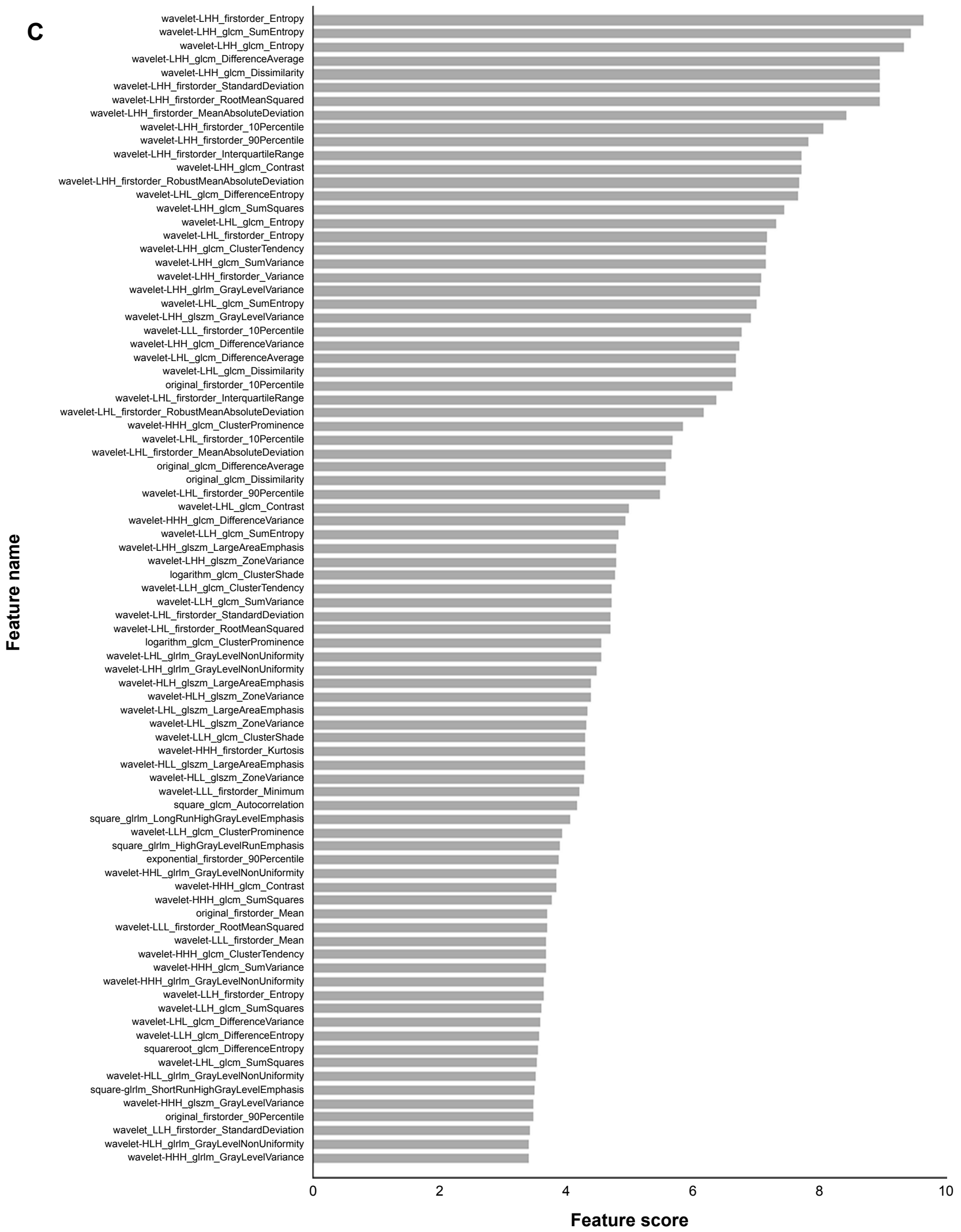

Figure 2 Feature selection.

Notes: (A) An example of manual delineation of primary lung lesions using CT image. The blue regions were the primary lung lesions manually delineated on the CT images by thoracic radiologists; The yellow region indicated the coordinates of the lesion regions; The number 2 indicated a total of 2 lesions for this patients. (B) Feature selection based on variance threshold $<0.8$. (C) Univariate analysis of the associations between the imaging phenotype and the treatment response. Twelve features with positive results are shown. 
glucocorticoid, with $30.4 \%$ and $36.4 \%$ patients administered cyclophosphamide in the training and validation groups, respectively. Treatment response rates in the training and validation groups were $38.5 \%$ and $36.4 \%$, respectively (Table 1). Thirty-four (11.0\%) patients in the training group and $20(18.7 \%)$ in the validation group experienced only symptom improvement or objective imaging response. Some patients in the training group $(n=129$ [41.7\%]) and $23(21.5 \%)$ in the validation group exhibited a treatment response at 3-6 months' follow-up; 121 (93.8\%) in the training group and $22(95.7 \%)$ in the validation group exhibited the same results compared with their treatment response evaluated at 2-6 weeks' follow-up. Fifty-three (17.1\%) patients in the training group and $33(30.8 \%)$ in the validation group underwent PFT before and 3-6 months after glucocorticoid treatment. Improvement in PFT was consistent with the treatment response criterion, which was 45 (84.9\%) and 27 (81.8\%).

In the training group, patients with treatment response had higher cyclophosphamide use and higher dosage of glucocorticoids. In the validation group, patients who exhibited a treatment response were younger and used a higher dosage of glucocorticoids (Table 2).

\section{Feature selection and radiomics signature building}

According to a variance threshold of $0.8,1,029$ texture features were reduced to 615 potential predictors based on 165 patients in the training cohort (Figure 2B). Univariate analysis of the associations between imaging phenotype and the treatment response identified 85 promising features (Figure 2C). The LASSO logistic regression model was then used to minimize the number of features. The best performance of LASSO regression was built using a penalty parameter $\alpha=1.7432$, as the mean square error was minimized. With nonzero coefficients, prognostic features were reduced to 11 (Figure $3 \mathrm{~A}-\mathrm{C}$, Table 3 ). The scale of the primary radiomics features confirmed that the derived conclusions were comprehensive and reliable, and the reduction protocol enabled the prediction models to avoid overfitting.

\section{Development of an individualized prediction model}

Models of RF and k-NN were built using radiomics features and response data from the training cohort, and their performance was evaluated in the validation cohort. ROC curves

Table I Patient characteristics in the training and testing cohorts

\begin{tabular}{|c|c|c|c|}
\hline \multirow[t]{2}{*}{ Characteristics } & \multirow{2}{*}{\begin{tabular}{|l} 
Training \\
$\mathrm{n}=309$
\end{tabular}} & \multirow{2}{*}{$\begin{array}{l}\text { Testing } \\
n=107\end{array}$} & \multirow[t]{2}{*}{$P$-value } \\
\hline & & & \\
\hline Gender & & & 0.671 \\
\hline Male & III (35.9\%) & $36(33.6 \%)$ & \\
\hline Female & $198(64.1 \%)$ & $71(66.4 \%)$ & \\
\hline Age (median, range, year) & $55(15-83)$ & $58(21-79)$ & 0.052 \\
\hline Smoke & $62(20.1 \%)$ & $18(16.8 \%)$ & 0.463 \\
\hline \multicolumn{4}{|l|}{ Symptoms } \\
\hline Fever & $75(24.3 \%)$ & $30(28.0 \%)$ & 0.44 \\
\hline Cough & $205(66.3 \%)$ & $60(56.1 \%)$ & 0.057 \\
\hline Sputum & $159(51.5 \%)$ & $44(41.1 \%)$ & 0.065 \\
\hline Dyspnea & $130(42.1 \%)$ & $38(35.6 \%)$ & 0.234 \\
\hline ICU admission & $60(19.4 \%)$ & $12(11.2 \%)$ & 0.053 \\
\hline Cyclophosphamide & 94 (30.4\%) & $39(36.4 \%)$ & 0.249 \\
\hline Glucocorticoids history & & & 0.667 \\
\hline Negative & 189 (6I.2\%) & $63(58.9 \%)$ & \\
\hline Positive & I 20 (38.8\%) & 44 (4I.I\%) & \\
\hline Glucocorticoid, high dosage & 69 (22.3\%) & $20(18.7 \%)$ & 0.429 \\
\hline Basic use of DMARDs & 40 (14.2\%) & $22(19.9 \%)$ & 0.057 \\
\hline \multicolumn{4}{|l|}{ Primary CTD } \\
\hline UCTD & $90(29.1 \%)$ & $4(3.7 \%)$ & \\
\hline DM & $88(28.5 \%)$ & $22(20.6 \%)$ & \\
\hline SLE & 38 (I2.3\%) & $4(3.70 \%)$ & \\
\hline RA & $21(6.80 \%)$ & $50(46.7 \%)$ & \\
\hline SSc & 36 (II.7\%) & $15(14.0 \%)$ & \\
\hline SS & $16(5.20 \%)$ & $4(3.70 \%)$ & \\
\hline MCTD & $20(6.50 \%)$ & $8(7.50 \%)$ & \\
\hline Response evaluation time (median, range, week) & $4(2-6)$ & $4(2-6)$ & 0.64 \\
\hline Treatment response & $119(38.5 \%)$ & $39(36.4 \%)$ & 0.705 \\
\hline
\end{tabular}

Abbreviations: CTD, connective tissue disease; DMARDs, disease-modifying anti-rheumatic drugs; ICU, intensive care unit; MCTD, mix connective tissue disease; SLE, systemic lupus erythematosus; RA, rheumatoid arthritis; SSc, systemic sclerosis; SS, Sjogren's syndrome; UCTD, undifferentiated connective tissue disease. 
Table 2 Characteristics of patients who responded or did not respond to treatment in the training and testing cohorts

\begin{tabular}{|c|c|c|c|c|c|c|}
\hline \multirow[t]{3}{*}{ Characteristics } & \multicolumn{2}{|c|}{ Training $(n=309)$} & \multirow[t]{3}{*}{$P$-value } & \multicolumn{2}{|c|}{ Testing $(n=107)$} & \multirow[t]{3}{*}{$P$-value } \\
\hline & Effective & Non-effective & & Effective & Non-effective & \\
\hline & $n=119$ & $n=190$ & & $n=39$ & $n=68$ & \\
\hline Gender & & & 0.161 & & & 0.634 \\
\hline Male & 37 (31.1\%) & 74 (38.9\%) & & $12(30.8 \%)$ & $24(35.3 \%)$ & \\
\hline Female & $88(68.9 \%)$ & $116(61.1 \%)$ & & $27(69.2 \%)$ & $44(64.7 \%)$ & \\
\hline Age (median, range, year) & $51(15-83)$ & $56(24-82)$ & 0.194 & $51(21-75)$ & $59(23-79)$ & 0.043 \\
\hline Smoke & $23(19.3 \%)$ & $39(20.5 \%)$ & 0.798 & $8(20.5 \%)$ & $10(14.7 \%)$ & 0.440 \\
\hline \multicolumn{7}{|l|}{ Symptoms } \\
\hline Fever & $31(26.1 \%)$ & $44(23.2 \%)$ & 0.564 & $12(30.8 \%)$ & $18(26.5 \%)$ & 0.634 \\
\hline Cough & $75(63.0 \%)$ & $130(68.4 \%)$ & 0.329 & $23(60.0 \%)$ & $37(54.4 \%)$ & 0.647 \\
\hline Sputum & $56(47.1 \%)$ & $103(54.2 \%)$ & 0.221 & $17(43.6 \%)$ & $27(39.7 \%)$ & 0.694 \\
\hline Dyspnea & $43(36.1 \%)$ & $87(45.8 \%)$ & 0.094 & $15(38.5 \%)$ & $23(33.8 \%)$ & 0.629 \\
\hline ICU admission & $19(16.0 \%)$ & $41(21.6 \%)$ & 0.225 & $2(5.10 \%)$ & $10(14.7 \%)$ & 0.204 \\
\hline Cyclophosphamide & $45(37.8 \%)$ & $49(25.8 \%)$ & 0.025 & $16(41.0 \%)$ & $23(33.8 \%)$ & 0.456 \\
\hline Glucocorticoids history & & & 0.504 & & & 0.694 \\
\hline Negative & 70 (58.8\%) & 119 (62.6\%) & & $22(56.4 \%)$ & $4 \mathrm{I}(60.3 \%)$ & \\
\hline Positive & $49(41.2 \%)$ & $71(37.4 \%)$ & & $17(43.6 \%)$ & $27(39.7 \%)$ & \\
\hline Glucocorticoid, high dosage & $39(32.8 \%)$ & $30(15.8 \%)$ & 0.001 & $12(30.8 \%)$ & $8(11.8 \%)$ & 0.015 \\
\hline Basic use of DMARDs & 14 (I I.8\%) & $26(13.7 \%)$ & 0.625 & $7(17.9 \%)$ & $15(22.1 \%)$ & 0.613 \\
\hline \multicolumn{7}{|l|}{ Primary CTD } \\
\hline UCTD & $29(24.3 \%)$ & 61 (32.1\%) & & 2 & 2 & \\
\hline DM & $31(26.0 \%)$ & $57(30.0 \%)$ & & 12 & 10 & \\
\hline SLE & $24(20.2 \%)$ & $14(7.4 \%)$ & & 2 & 2 & \\
\hline RA & $10(8.4 \%)$ & II (5.8\%) & & 15 & 35 & \\
\hline SSc & II (9.2\%) & $25(13.2 \%)$ & & 2 & 13 & \\
\hline SS & $6(5.0 \%)$ & $10(5.3 \%)$ & & 2 & 2 & \\
\hline MCTD & $8(6.7 \%)$ & $12(6.3 \%)$ & & 4 & 4 & \\
\hline Response evaluation time (median, range, week) & $4(2-6)$ & $4(2-6)$ & 0.819 & $4(2-6)$ & $4(2-6)$ & 0.370 \\
\hline
\end{tabular}

Abbreviations: CTD, connective tissue disease; DMARDs, disease-modifying anti-rheumatic drugs; ICU, intensive care unit; MCTD, mix CTD; SLE, systemic lupus erythematosus; RA, rheumatoid arthritis; SSc, systemic sclerosis; SS, Sjogren's syndrome; UCTD, undifferentiated CTD.

indicated that the AUCs were 0.66 in RF models and 0.61 in $\mathrm{k}-\mathrm{NN}$ model (Figure 4A and B). The clinical data were then integrated into the training $\mathrm{RF}$ and k-NN models, which yielded AUCs of 0.71 and 0.61 (Figure 4C and D). With the addition of clinical data, the prediction performance of the RF model was improved (sensitivity $=0.897$ ). The result computed from the logistic regression of radiomics features and clinical data is shown in the nomogram (Figure 5A). The calibration curve for the probability of treatment response in the training and validation cohorts demonstrated good agreement between prediction and observation (Figure 5B and C).

\section{Clinical use}

Decision curve analysis for the radiomics and clinical data nomogram (Figure 5D) demonstrated that if the threshold probability of a patient was between $20 \%$ and $60 \%$, using the radiomics nomogram to predict treatment response added more benefit than either the treat-all-patients scheme or the treat-none scheme. Because the response rate was $38.5 \%$ and $36.4 \%$ in the training and validation groups, respectively, it could be assumed that glucocorticoid would be beneficial if the predicted response rate was $50 \%-60 \%$ for an individual according to the radiomics and clinical data nomogram.

\section{Discussion}

CTD-ILD is highly heterogeneous, ${ }^{1}$ and treatment strategies have been controversial given the lack of widely acknowledged remedies. Although glucocorticoids have been widely used to treat CTD-ILD, there is a lack of supportive, clinical trial-based evidence. ${ }^{5,6}$ This situation may partially be caused by low treatment response rates, a high incidence of side effects, and difficulty in disease stratification. ${ }^{17}$ Part of CTDILD is considered to be sensitive to glucocorticoid, such as acute exacerbation of chronic lung injury. ${ }^{7}$ The early use of glucocorticoids may be of some help for prognosis. However, long-term glucocorticoid treatment may lead to infection, osteoporosis, gastrointestinal bleeding, and other severe side effects. Nevertheless, the timing of glucocorticoid therapy in patients with ILD remains a clinical puzzle. ${ }^{18} \mathrm{~A}$ solution to this issue may involve identifying patients who are sensitive to glucocorticoid treatment. In the present study, the treatment response to glucocorticoid was $36.4 \%-38.5 \%$, which was disappointing given its potential side effects. However, among patients who were predicted to be sensitive to glucocorticoid using the radiomics approach, the treatment response increased to $68.5 \%$. Thus, the present study identified a group of patients with a doubled response rate to glucocorticoid. 
A

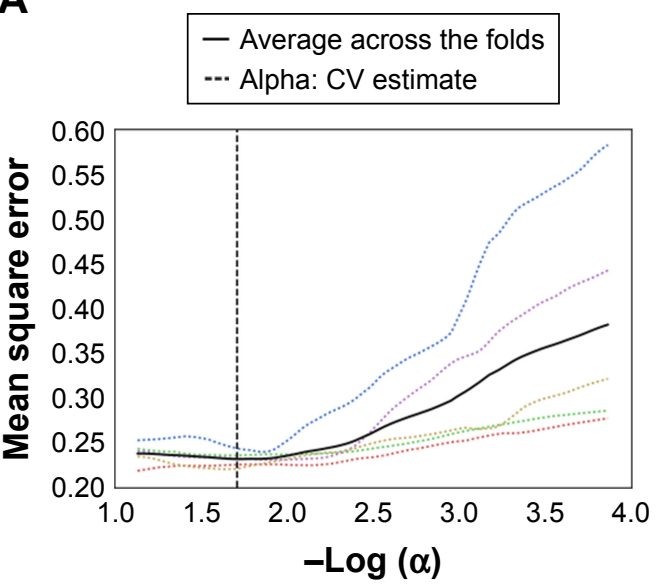

B

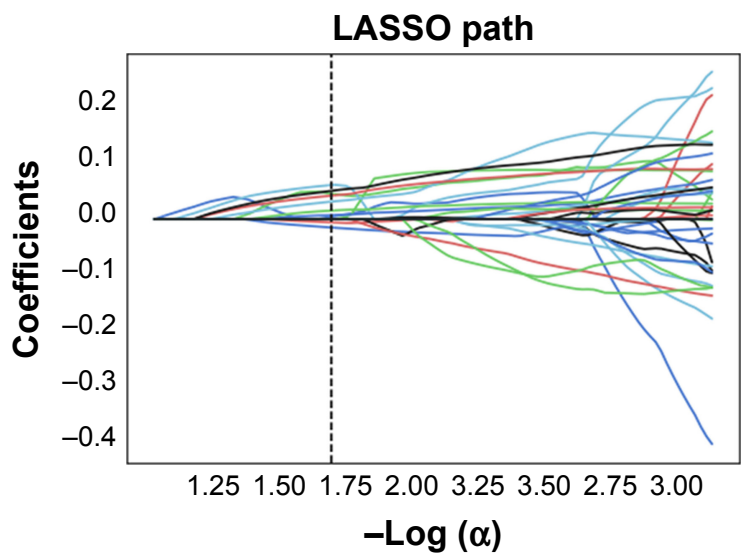

C

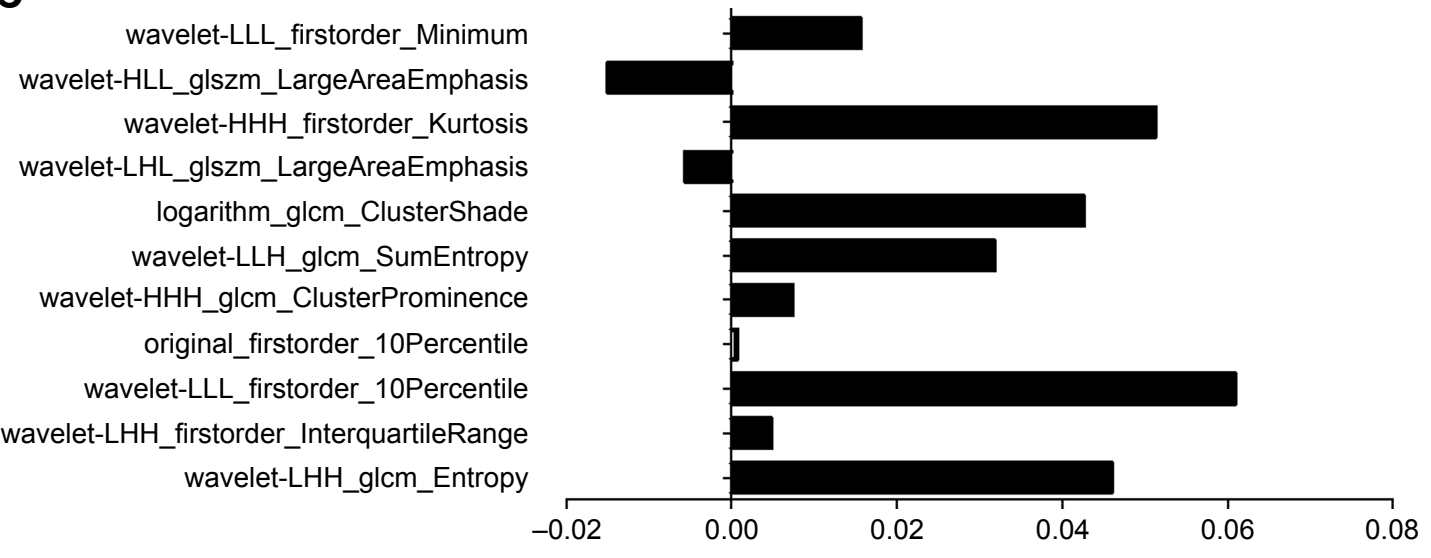

Coefficients in the LASSO model

Figure 3 Feature selection using the LASSO binary logistic model.

Notes: (A) Mean square error on each fold in fivefold cross-validation method. Vertical dotted line was drawn at the minimum mean square error of average. The optimal penalty parameter alpha was obtained based on the line. (B) LASSO coefficient solution path of the eleven features. A coefficient profile plot was produced according to the $\log$ (alpha) sequence. Vertical line was drawn at the value selected using fivefold cross-validation, where optimal alpha resulted in seven nonzero coefficients. (C) Coefficients in the LASSO model of the eleven features.

Abbreviation: LASSO, least absolute shrinkage and selection operator.

CTD-ILD can be stratified according to multiple criteria including primary disease, histopathological pattern of lesions, and a few classic findings on HRCT. ${ }^{19}$ However, none of these classifications aim to predict treatment response.

Table 3 LASSO coefficient profiles of the eleven features

\begin{tabular}{l|l}
\hline Features & Coefficients \\
\hline wavelet-LHH_glcm_Entropy & 0.045928 \\
wavelet-LHH_firstorder_InterquartileRange & 0.004803 \\
wavelet-LLL_firstorder_IOPercentile & 0.06097 I \\
original_firstorder_IOPercentile & 0.000646 \\
wavelet-HHH_glcm_ClusterProminence & 0.00737 I \\
wavelet-LLH_glcm_SumEntropy & 0.031787 \\
logarithm_glcm_ClusterShade & 0.04257 I \\
wavelet-LHL_glszm_LargeAreaEmphasis & -0.00564 \\
wavelet-HHH_firstorder_Kurtosis & 0.051242 \\
wavelet-HLL_glszm_LargeAreaEmphasis & -0.01495 \\
wavelet-LLL_firstorder_Minimum & 0.015582 \\
\hline
\end{tabular}

Abbreviation: LASSO, least absolute shrinkage and selection operator.
In ILD patients, previous studies investigating glucocorticoid treatment suggested that nonspecific interstitial pneumonia was sensitive to treatment and that usual interstitial pneumonia was refractory. ${ }^{4,20}$ However, pathological biopsy is difficult to obtain from these patients. According to data in our nomogram, diagnosis of CTD contributed little to the treatment response, and radiomics played the dominant role in treatment efficacy. Our findings suggest that CTD-ILD with stable extra-thoracic manifestations should be treated as a whole for glucocorticoid-based remedies. Previous studies investigating glucocorticoid treatment of ILD were mainly based on small sample sizes, or the treatment strategies were based on individual decisions. ${ }^{5,21,22}$ Thus, it is difficult to directly compare treatment responses among studies. Treatment strategies involving glucocorticoid have not been standardized according to consensus. ${ }^{17}$ In the present study, which had a relatively adequate sample size, we found that 

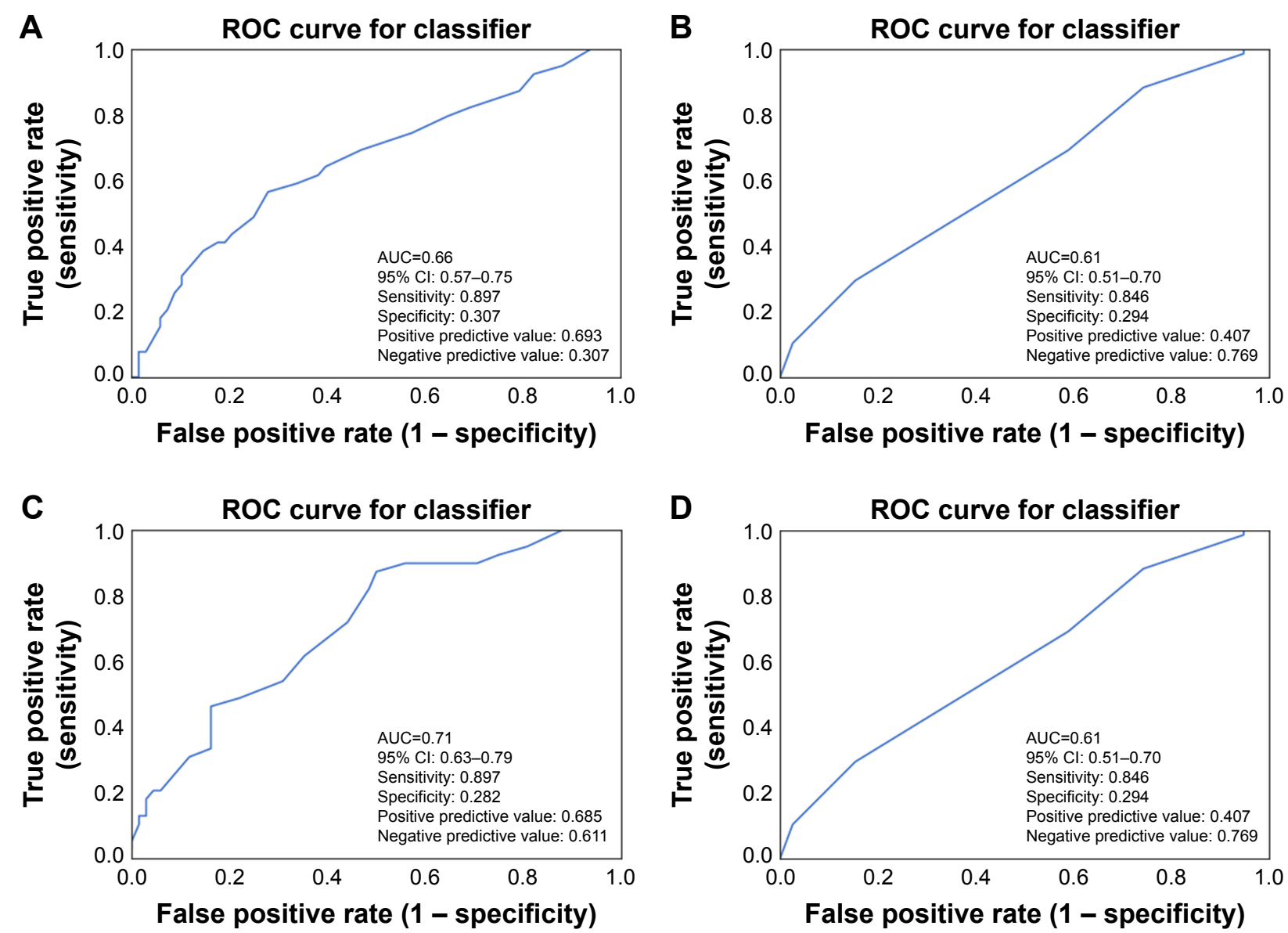

Figure 4 ROC curves for machine learning of radiomics to predict treatment response.

Notes: ROC analysis of radiomics to predict treatment response by $\operatorname{RF}(\mathbf{A})$ and k-NN (B) models. ROC analysis of radiomics and clinical data to predict treatment response by RF (C) and k-NN (D) models.

Abbreviations: AUC, area under the curve; k-NN, k-nearest neighbors; RF, random forest; ROC, receiver-operating characteristic.

high doses of glucocorticoid (pulsed intravenous methylprednisolone [initial dose of $1 \mathrm{mg} / \mathrm{kg} /$ day of prednisolone or $0.8 \mathrm{mg} / \mathrm{kg} /$ day of methylprednisolone]) with cyclophosphamide may be a promising combination in the treatment of CTD-ILD.

HRCT is a noninvasive technology for the diagnosis of ILD. Many studies have reported that HRCT is very important in determining the extent and severity of ILD. ${ }^{23-25}$ The distribution and manifestation of HRCT lesions may be related to the pathological type of ILD. ${ }^{23}$ Patterns of ILD have been characterized by a few HRCT manifestations, including ground-glass opacities, interlobular septal thickening, and grid-like, honeycomb-like lesions. ${ }^{13,26}$ However, a patient may present all types of lesions. Joseph Jacob et al and other studies found that HRCT score was also associated with prognosis in CTD-ILD patients. ${ }^{24}$ However, these scores are qualitative instead of quantitative, which limit their accuracy. Radiomics ${ }^{10,11}$ converts medical images into highdimensional, mineable data via high-throughput extraction of quantitative features, followed by subsequent data analysis for decision support. Results of the present study suggest that radiomics could be used to stratify CTD-ILD patients into glucocorticoid-sensitive and non-sensitive patients.

\section{Limitations}

The present study lacked survival and PFT data, which were designated to be the ultimate goal in the treatment of ILD. Only $53(17.1 \%)$ patients in the training group and 33 (30.8\%) in the validation group underwent PFT before and after glucocorticoid administration. We found that improvement in PFT was consistent with our treatment response criterion, which was 45 (84.9\%) and 27 (81.8\%). Although PFT was not included in our study, the accuracy of our treatment response evaluation should reflect PFT evolution. Moreover, the majority of patients did not undergo long-term treatment response assessment due to the retrospective nature of the present investigation. Moreover, the present study mainly involved Chinese subjects. Although the present investigation was a multicenter study, the results cannot be generalized to other populations. Thus, more multicenter studies and global 

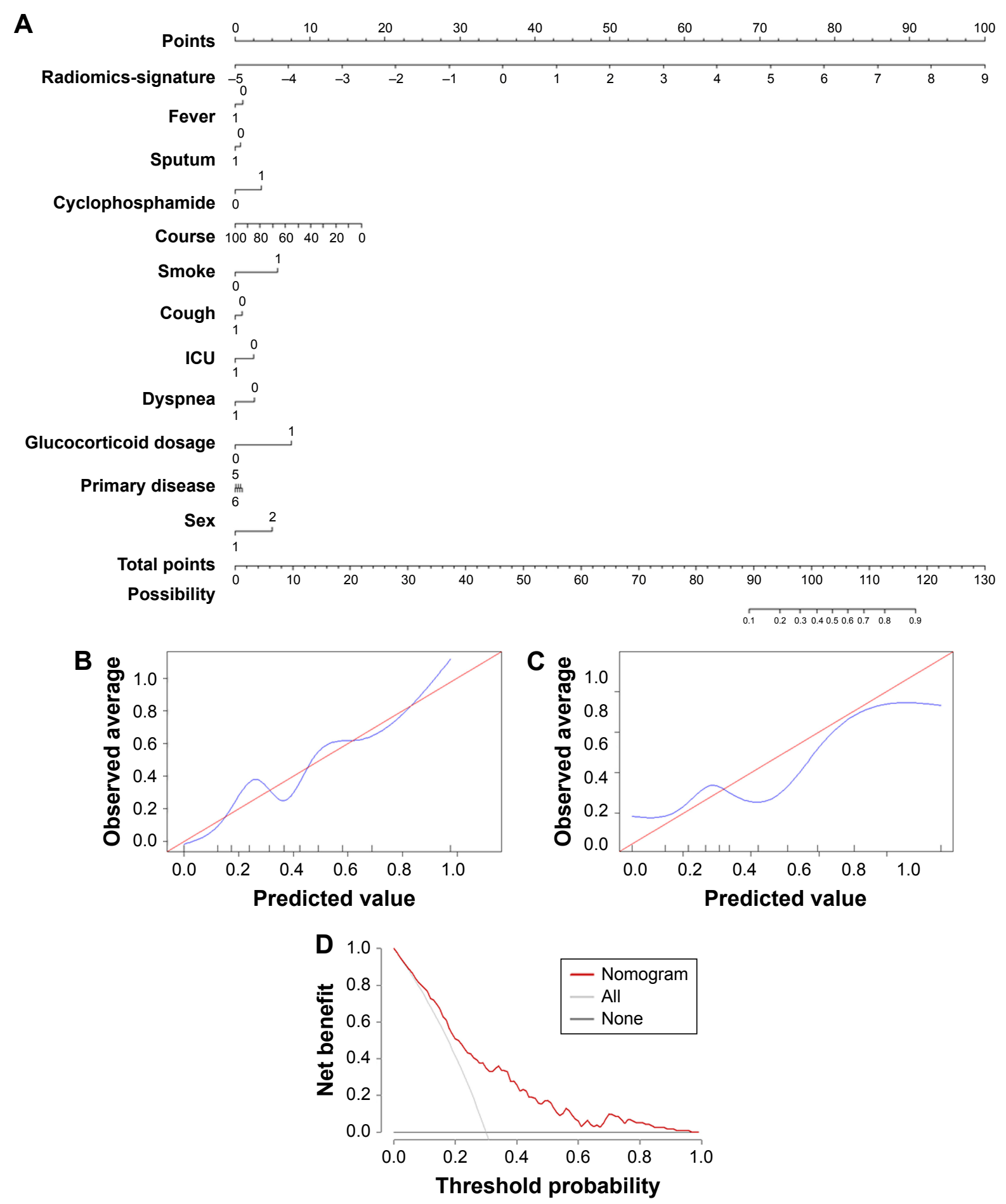

Figure 5 Developed radiomics nomogram

Notes: (A) The radiomics nomogram was developed in the training group with the radiomics signature and clinical data to predict treatment response. (B) Calibration curves of the radiomics and clinical data nomogram in the training group. (C) Calibration curve of the radiomics and clinical data nomogram in the validation group. (D) Decision curve analysis for the radiomics and clinical data nomogram. The $y$-axis measures the net benefit. The gray line represents the assumption that all patients respond to treatment. Black line represents the assumption that no patients respond to glucocorticoid. The net benefit was calculated by subtracting the proportion of all false-positive patients from the true-positive proportion, weighting by the relative harm of forgoing treatment compared with the negative consequences of an unnecessary treatment. Abbreviation: ICU, intensive care unit.

collaborations are needed to determine a precise predictive tool for treatment selection.

In summary, the present study built a predictive model using radiomics and clinical data to stratify CTD-ILD patients into glucocorticoid-sensitive and non-sensitive subgroups. In the sensitive group, the treatment response was double that of the unselected population. Future clinical trials investigating radiomics-guided glucocorticoid treatment are warranted. 


\section{Data sharing statement}

Both datasets are available from the corresponding author. There are no additional unpublished data.

\section{Acknowledgments}

This study was supported by National Natural Science Foundation of China (81700645 and 31600710), Natural Science Foundation of Guangdong (No 2017A030313537 and 2016A030313302), Guangzhou science and technology plan (201709010030), the Scientific Research Project of Guangzhou Municipal Universities (1201630019) and Guangzhou industry university research cooperative innovation major project (2014y2-00510), and Guangdong science and technology project in the field of social development (2013B021400002).

\section{Author contributions}

Tian-Tuo Zhang and Xing Li designed this study, interpreted the data, and are the guarantor of the paper, taking responsibility for the integrity of the work as a whole, from inception to publication of the article. Xing Li and Yan-Fang Xing wrote the manuscript. Ding-Yun Feng, Yu-Qi Zhou, Chuang-Feng Li, Jie Qin, Yue-Fei Guo, and Tian-Tuo Zhang evaluated the patients. Chuang-Feng Li, Jie Qin, and Yue-Fei Guo defined the lesion. Qing Lv, Yan-Fang Xing, Nan Jiang, Hai-Tao Hu, Jie Chen, Liang-Hong Yin, and Xing-Hua Guo collected the patients. Xing Li, Xing Yan-Fang, and Ding-Yun Feng conducted statistical analysis. Jie Dong and Chencui Huang conducted the radiomics analysis. All authors contributed toward data analysis, drafting and revising the paper and agree to be accountable for all aspects of the work.

\section{Disclosure}

The authors report no conflicts of interest in this work.

\section{References}

1. Ferri C, Manfredi A, Sebastiani M, et al. Interstitial pneumonia with autoimmune features and undifferentiated connective tissue disease: Our interdisciplinary rheumatology-pneumology experience, and review of the literature. Autoimmun Rev. 2016;15(1):61-70.

2. Storme M, Semionov A, Assayag D, et al. Estimating the incidence of interstitial lung diseases in the Cree of Eeyou Istchee, northern Québec. PLoS One. 2017;12(9):e0184548.

3. Maher TM. Immunosuppression for connective tissue disease-related pulmonary disease. Semin Respir Crit Care Med. 2014;35(2):265-273.

4. Migita K, Arai T, Jiuchi Y, et al. Predictors of mortality in patients with interstitial lung disease treated with corticosteroids: results from a cohort study. Medicine. 2014;93(26):e175.

5. Sharma N, Putman MS, Vij R, Strek ME, Dua A. Myositis-associated Interstitial Lung Disease: Predictors of Failure of Conventional Treatment and Response to Tacrolimus in a US Cohort. J Rheumatol. 2017; 44(11):1612-1618.
6. Go DJ, Park JK, Kang EH, et al. Survival benefit associated with early cyclosporine treatment for dermatomyositis-associated interstitial lung disease. Rheumatol Int. 2016;36(1):125-131.

7. Robles-Perez A, Molina-Molina M. Treatment Considerations of Lung Involvement in Rheumatologic Disease. Respiration. 2015;90(4): 265-274.

8. Rice JB, White AG, Scarpati LM, Wan G, Nelson WW. Long-term Systemic Corticosteroid Exposure: A Systematic Literature Review. Clin Ther. 2017;39(11):2216-2229.

9. Gillies RJ, Kinahan PE, Hricak H. Radiomics: Images Are More than Pictures, They Are Data. Radiology. 2016;278(2):563-577.

10. Aerts HJ, Velazquez ER, Leijenaar RT, et al. Decoding tumour phenotype by noninvasive imaging using a quantitative radiomics approach. Nat Commun. 2014;5:4006.

11. Scrivener M, de Jong EEC, van Timmeren JE, Pieters T, Ghaye B, Geets X. Radiomics applied to lung cancer: a review. Transl Cancer Res. 2016;5(4):398-409.

12. Travis WD, Costabel U, Hansell DM, et al. An official American Thoracic Society/European Respiratory Society statement: Update of the international multidisciplinary classification of the idiopathic interstitial pneumonias. Am J Respir Crit Care Med. 2013;188(6):733-748.

13. Hu Y, Wang LS, Wei YR, et al. Clinical Characteristics of Connective Tissue Disease-Associated Interstitial Lung Disease in 1,044 Chinese Patients. Chest. 2016;149(1):201-208.

14. Miller MR, Hankinson J, Brusasco V, et al. Standardisation of spirometry. Eur Respir J. 2005;26(2):319-338.

15. Huang YQ, Liang CH, He L, et al. Development and Validation of a Radiomics Nomogram for Preoperative Prediction of Lymph Node Metastasis in Colorectal Cancer. J Clin Oncol. 2016;34(18): 2157-2164.

16. Rios Velazquez E, Parmar C, Liu Y, et al. Somatic Mutations Drive Distinct Imaging Phenotypes in Lung Cancer. Cancer Res. 2017; 77(14):3922-3930.

17. Lee JS, Fischer A. Current and emerging treatment options for interstitial lung disease in patients with rheumatic disease. Expert Rev Clin Immunol. 2016;12(5):509-520.

18. Behr J, Neuser P, Prasse A, et al. Exploring efficacy and safety of oral Pirfenidone for progressive, non-IPF lung fibrosis (RELIEF) - a randomized, double-blind, placebo-controlled, parallel group, multi-center, phase II trial. BMC Pulm Med. 2017;17(1):122.

19. Tsuchiya Y, Fischer A, Solomon JJ, Lynch DA. Connective Tissue Disease-related Thoracic Disease. Clin Chest Med. 2015;36(2): 283-297.

20. Li X, Chen $\mathrm{C}, \mathrm{Xu}$ J, et al. Nonspecific interstitial pneumonia and usual interstitial pneumonia: comparison of the clinicopathologic features and prognosis. J Thorac Dis. 2014;6(10):1476-1481.

21. Reina D, Roig Vilaseca D, Torrente-Segarra V, et al. Sjögren's syndrome-associated interstitial lung disease: A multicenter study. Reumatol Clin. 2016;12(4):201-205.

22. Adler S, Huscher D, Siegert E, et al. Systemic sclerosis associated interstitial lung disease - individualized immunosuppressive therapy and course of lung function: results of the EUSTAR group. Arthritis Res Ther. 2018;20(1):17.

23. Jun S, Park B, Seo JB, Lee S, Kim N. Development of a ComputerAided Differential Diagnosis System to Distinguish Between Usual Interstitial Pneumonia and Non-specific Interstitial Pneumonia Using Texture- and Shape-Based Hierarchical Classifiers on HRCT Images. J Digit Imaging. 2018;31(2):235-244.

24. Jacob J, Bartholmai BJ, Rajagopalan S, et al. Evaluation of computerbased computer tomography stratification against outcome models in connective tissue disease-related interstitial lung disease: a patient outcome study. BMC Med. 2016;14(1):190.

25. Toyoda Y, Hanibuchi M, Kishi J, et al. Clinical features and outcome of acute exacerbation of interstitial pneumonia associated with connective tissue disease. J Med Invest. 2016;63(3-4):294-299.

26. Vij R, Noth I, Strek ME. Autoimmune-featured interstitial lung disease: a distinct entity. Chest. 2011;140(5):1292-1299. 


\section{Publish your work in this journal}

Therapeutics and Clinical Risk Management is an international, peerreviewed journal of clinical therapeutics and risk management, focusing on concise rapid reporting of clinical studies in all therapeutic areas, outcomes, safety, and programs for the effective, safe, and sustained use of medicines. This journal is indexed on PubMed Central, CAS,

EMBase, Scopus and the Elsevier Bibliographic databases. The manuscript management system is completely online and includes a very quick and fair peer-review system, which is all easy to use. Visit http://www.dovepress.com/testimonials.php to read real quotes from published authors.

Submit your manuscript here: http://www.dovepress.com/therapeutics-and-clinical-risk-management-journal 